\title{
Typ-I-Allergie: den Krankheitsverlauf modulieren
}

Die zentrale Bedeutung, die man heute den T-Zellen bei IgE-vermittelten allergischen Erkrankungen beimi $\beta$ t, gibt auch die Richtung an, in der zur Zeit an innovativen Therapiekonzepten gearbeitet wird. Therapieziel ist es, das Fortschreiten der Erkankung zu verhindern und damit auch den charakteristischen Manifestationswechsel im Laufe der Allergiker-Karriere zu unterbrechen.

$\mathrm{D}$ ie Möglichkeiten, die heute zur Intervention bei allergischen Erkrankungen zur Verfügung stehen, setzen relativ weit am Ende der Kaskade ein, welche zwischen Allergen-Präsentation und Auslösung der Sofort- sowie Spätreaktion an der Zielzelle abläuft: an der Mastzelle, wo die Stabilisatoren Cromoglycinsäure und Nedocromil die Ausschüttung deren Mediatoren verhindern, an den Rezeptoren, wo Antihistaminika die Histamin-vermittelten Reaktionen unterbinden, an der glatten Muskulatur, wo Beta-2-Sympathomimetika bzw. Theophyllin dem Bronchospasmus entgegenwirken, sowie an den Entzündungszellen, wo Glukokortikoide die Spätreaktion modulieren.

Darin, daß in Kürze noch weitere Mediatorstoffe antagonisierbar sein werden, sieht U. Wahn, Berlin, keinen entscheidenden Fortschritt. Schließlich sei angesichts des komplexen Netzwerks von Mediatoren kaum anzunehmen, daß die Ausschaltung eines einzigen davon beschwerdefrei macht! Wesentlich spannender sei es, weiter unten auf der Stufenleiter der spezifischen Immunantwort anzusetzen, z.B. an der Stelle der T-Helferzellen-Differenzierung, wo man derzeit den Wirkmechanismus der spezifischen Immuntherapie ansiedelt. Seit man über die wichtige modulierende
Rolle der T-Lymphozyten-Subpopulationen dazugelernt hat und die in diesem Zusammenhang entscheidende Funktion der Antigen-Präsentation kennt, ist man dabei, an solch frühen Interventionen $\mathrm{zu}$ arbeiten.

\section{Vielversprechende Perspektiven}

$\mathrm{Zu}$ den in Entwicklung befindlichen Ansätzen gehören z.B. Versuche, im Rahmen einer Immuntherapie solche Peptide anzubieten, die den T-Lymphozyten nicht aktivieren, vielmehr in eine Art „Dauerschlaf“ versetzen, also anerg machen.

Mit anderen Konzepten versucht man, ein Schlüssel-Zytokin der TH2Lymphozyten, welches die B-Zellen aktiviert und zur IgE-Produktion veranlaßt, nämlich IL-4, zu antagonisieren. In vitro hat sich die Idee mit dem IL-4-Rezeptor bereits bewährt: Am Institut für Klinische Chemie und Biochemie des Virchow-Klinikums, Berlin, kultivierte man Lymphozyten von Patienten mit ausgeprägter Milbenallergie. Durch Inkubation mit einem (gentechnologisch herstellbaren) löslichen Rezeptor für IL-4 wurde die Produktion spezifischer IgE-Antikörper massiv unterdrückt.

Bis solche Modulationen der Immunantwort jedoch beim Menschen möglich sind, bleibt die Immuntherapie eine wichtige Option in der Therapie IgE-vermittelter allergischer Erkrankungen - und einziges etabliertes Verfahren, das kausale Behandlung verspricht.

\section{Langzeit- und präventive Effekte der Immuntherapie}

Daß der Effekt einer erfolgreichen Immuntherapie auch nach Therapieende noch über mehrere Jahre anhält, konnten S.R. Durham, London, et al. in einer prospektiven kontrollierten Langzeitstudie nachweisen. Bei Hausstaubmilbenallergie erwies sich kürzlich die spezifische Immuntherapie nicht nur in bezug auf Allergen-induzierte Symptomatik und Medikamentenverbrauch als hilfreich: Zusätzlich, so konnte W.J. Pichler, Bern, zeigen, ging unter der Behandlung auch die nasale oder bronchiale - Hyperreaktivität signifikant zurück - jene Beschwerden also, unter denen die Patienten mit perennialen Atemwegserkrankungen vorrangig leiden.

Das präventive Potential dieser Therapieform, ihr Vermögen, bei Allergien der oberen Atemwege dem gefürchteten Etagenwechsel vorzubeugen, wird derzeit in der multizentrischen PAT-Studie (Preventive Allergy Treatment) untersucht, an der auch die Allergologen des Berliner Virchow-Klinikums teilnehmen. Endpunkt ist das Auftreten von Asthma bei 210 Kindern mit allergischer Rhinokonjunktivitis zwischen 6 und 13 Jahren. Die Patienten wurden randomisiert entweder der Gruppe mit spezifischer Immuntherapie (mit ALKdepot SQ) zugeteilt oder der Kontrollgruppe, in der nur optimal medikamentös versorgt wird; diese Medikation ist in beiden Gruppen gleich.

Nach ersten Auswertungen der auf zehn Jahre angelegten Studie kann das Risiko einer Asthmaentwicklung bei Pollenallergikern in der Tat durch Hyposensibilisierung verringert werden. Nach drei Jahren bereits war nach einer Analyse der schwedischen und finnischen Daten ein signifikanter Vorteil für die aktiv Behandelten gefunden worden.

(wpa)

\footnotetext{
4. Internationales Allergie-Symposium, Berlin, 20.-21.6.1997 (Veranstalter: Scherax Arzneimittel GmbH, Hamburg).
} 